\title{
THE PROTECTION OF THE MARRIAGE BOND AND POLYGAMY IN THE SPANISH MATRIMONIAL SYSTEM
}

\section{LA PROTEZIONE DEL VINCOLO MATRIMONIALE E LA POLIGAMIA NEL SISTEMA MATRIMONIALE SPAGNOLO}

\begin{abstract}
In Spanish Law, the constitutional public order, ruled by Spanish Constitution and by Spanish Religious Freedom Act, has the consequence that it's not possible to marry a second marriage if the first one has not been dissolved before, and this is not able to do not even when it there is a religious motivation. So, monogamic marriage is recognized in Spanish Law, both in Civil as in Canonic Law, which Canonic marriage is recognized by Spanish matrimonial system. This protection is done by the establishment of prohibitions for contract a new marriage while the first one has not been dissolved, and, also by the crime of bigamy. However, it has been admitted by Spanish Supreme Court, some legal consequences from a polygamic marriage what it could be the way for a further admission of polygamic marriage in the future.

This work has been done inside the Proyecto I+D+I DER2015-65840-R 'Diversidad y Convivencia: los derechos humanos como guía de acción', del Programa Estatal de Investigación, Desarrollo e Innovación orientada a los retos de la sociedad, del Ministerio de Economía y Competitividad.
\end{abstract}

\section{RiASSUNTO}

Nell'ordinamento giuridico spagnolo, l'ordine pubblico costituzionale, regolato nella Costituzione Spagnola ed anche nella legge organica di libertà religiosa, attua in modo che non si possa contrarre un secondo matrimonio mentre ci sia in vigore il primo matrimonio; e non si può fare questo neppure per motivi religiosi. Così, la monogamia matrimoniale viene protetta nel diritto spagnolo, tanto nel diritto civile spagnolo come nel diritto matrimoniale canonico, il cui è ammesso nel sistema 
matrimoniale spagnolo attuale. Questa protezione viene data tanto per mezzo di impedimenti o divieti per contrarre un nuovo matrimonio mentre non sia dissolto il primo matrimonio, come per l'esistenza del reato di bigamia. Nonnostante questo, la Corte Suprema Spagnola ha già ammesso delle conseguenze giuridiche derivate di un matrimonio poligamico, e questo potrebbe supporre una via per l'ammissione più ampia del matrimonio poligamico nel futuro.

Questo lavoro è stato fatto nel Proyecto I+D+I DER2015-65840-R 'Diversidad y Convivencia: los derechos humanos como guía de acción', del Programa Estatal de Investigación, Desarrollo e Innovación orientada a los retos de la sociedad, del Ministerio de Economía y Competitividad.

KeYwORDs: marriage, poligamy, public order, Islamic law, Spanish law

Parole Chiave: matrimonio, poligamia, ordine pubblico, diritto Islamico, diritto Spagnolo

\section{INTRODUZIONE: ORDINE PUBBLICO E SISTEMA MATRIMONIALE}

a) Lordine público nella legislazione spagnola

Lordine pubblico (in spagnolo, „orden público”) viene inserito nella Costituzione spagnola dal 1978, come una limitazione per l'agevolazione del diritto di libertà religiosa. Concretamente, la sua ammissione costituzionale si trova nell'articolo 16.1, il cui dice che, è garantita la libertà idelologica, religiosa e diculto degli individui e delle comunità senzaltra limitazione, nelle sue manifestazioni, che quella necessaria per mantenere l'ordine pubblico protetto dalla legge".

Lo sviluppo normativo della cosidetta previssione costituzionale cè nell'articolo 3.1 della LOLR (Legge Organica sulla Libertà Religiosa), Legge 7/1985, del 5 luglio 1980, che recita: „'esercizio dei diritti compressi nella libertà religiosa e di culto, ha l'unico limite nella protezione dei diritti altrui nell'esercizio delle sue libertà pubbliche e diritti fondamentali, anzicchè la sicurezza, la salute e la moralità pubblica, che sono gli elementi costitutivi dell'ordine pubblico protetto dalla legge nel contesto d'una società democratica".

In un primo sguardo si può affermare che l'ordine pubblico spagnolo previene e impedisce che la libertà religiosa sia una scusa per poter fare "qualsiasi cosa”. In altre parole: non tutto quello che sia permesso per una religione, o per il diritto religioso di essa, sarà permesso di fare in Spagna, come accade con la poligamia islamica. 
In questo senso, la poligamia sarebbe contraria all'ordine pubblico spagnolo nel suo senso di moralità pubblica ed anche per contravenire dei diritti fondamentali come quello dell'uguaglianza, che in materia matrimoniale ci deve essere fra $\mathrm{i}$ coniugi, dato che mai potrà dirsi che abbia uguaglianza in una relazione in cui ci sia un único uomo e che le mogli possano essere fino a quattro,

In conseguenza, la monogamia matrimoniale viene protetta dallordinamento giuridico spagnolo, invece, la poligamia non: essa sarebbe, d'inizio, non ammessa dallordinamento spagnolo, e per tanto, inefficace per essere respinta dallordine pubblico spagnolo (Rodríguez García, 2001, p. 745).

Questa realtà non è stata una invenzione dellordinamento giuridico spagnolo, perchè la monogamia céra indubbiamente già protetta dal diritto matrimoniale romano, che rifiutava la poligamia che esisteva in altri ordinamenti giuridici d’allora (Navarro-Valls, 1994, p. 22). È per questo che non si può dire che la monogamia abbia stato una aportazione giuridica del cristianesimo allá concezione occidentale del matrimonio (Gaudemet, 1993, pp. 53 e 69).

Nonnostante, nel diritto romano, la monogamia non aveva lo stesso profilo giuridico che ebbe il matrimonio nel medioevo, allora sì con maggiore tracce cristiane. Così, c'erano due motivi per cui nel matrimonio romano non c'entrava, d'inizio, il reato di bigamia: d'una parte, perchè la convivenza simultanea di due persone supponeva inmediatamente la mancanza d'onorabilità soziale che si voleva por contrarre matrimonio, e d'altra parte, dato che per il diritto romano il consenso matrimoniale non si considerava come "pattizio" ma come "consenso continuato", il fatto che un congiuge lasciassi l'altro per iniziare la convivenza con altra persona supponeva la dissoluzione del primo matrimonio e l'inizio del nuovo (Vega Gutiérrez, 1997, p. 128). Nonnostante, quando il cristianesimo modificò la visione del consenso matrimoniale e lo trasformò da continuato a patticio, la bigamia diviene un reato autonomo, differente d'altri come lo strupro o l'adulterio. Con questa nuova concezione del consenso matrimoniale, d'ispirazione cristiana, se si lasciava il congiuge per iniziare una nuova convivenza con un'altra persona, questo fatto non dissolveva il primo matrimonio, perchè per dissolverlo si voleva un atto di consenso contrario, con volontà specifica di dissolvere questo, e se l'atto concreto di dissoluzione non era fatto prima di dare il nuovo consenso, questo nuovo consenso supponeva unattacco 
alla monogamia matrimoniale, un reato di bigamia (Vega Gutiérrez, 1997, pp. 135-136).

b) Il sistema matrimoniale spagnolo

Il rifiuto della poligamia nel diritto spagnolo viene rafforzata dal suo sistema matrimoniale, che è un sistema misto dove si abbina la facoltà di scelta iniziale tra due tipi diversi di matrimonio, quello canonico e quello civile, ognuno regolato dalle sue proprie norme (questa scelta si chiama „sistema matrimoniale facoltativo di tipo latino”), e una seconda scelta, nel contesto del matrimonio civile, proprio nel momento della sua celebrazione, momento in cui si può scegliere tra la forma civile pure o quella forma religiosa delle religioni che hanno riuscito a firmare una intesa con lo Stato, comè il caso della religione islámica (accanto ai protestante e agli ebrei), o al meno, godono del "notorio arraigo" (radicamento notabile) dichiarato per lo Stato come requisito per firmare una intesa. In questa situazione si trovano la forma matrimoniale dei testimoni di Geova, i mormoni, gli ortodossi e i buddhisti.

In modo diverso a come accade col matrimonio canonico, dove cè una ammissione totale d'unaparte dell'ordinamento giuridico della Chiesa cattolica, quella matrimoniale, compressi i motivi d'incapacità e i divieti matrimoniali canonici (chiamati impedimenti), nel caso del diritto matrimoniale islámico soltanto viene riconosciuta la sua forma di celebrazione, non il resto di norme islamiche sulla capacità o abilità dei contraenti. In conseguenza, accanto all'ordine pubblico, questo è unaltro motivo per cui la poligamia non sia riconosciuta dal diritto spagnolo,.

In altre parole, la regolazione matrimoniale canonica viene ammessa in tutta la sua pienezza, tanto quella che fa riferimento allá celebrazione del vincolo come i requisiti per poter fare la celebrazione dai coniugi, la sua abilità consensuale e i divieti o impedimenti. In modo parallelo, il matrimonio canonico è monogámico, come quello regolato dal diritto civile spagnolo. Tuttavia, la legge islámica in materia matrimoniale, che ammette la poligamia, soltanto viene ammessa per quanto riguarda la forma di celebrazione del matrimonio, latto d'esprimere il consenso matrimoniale dai coniugi, e per il resto (capacità consensuael e divieti matrimoniali) si applica la legge civile spagnola 
Adesso è giusto vedere questa differenza nel suo contesto legale. Così, in primo luogo, dobbiamo fare riferimento all'articolo VI.1. dell'Accordo di 3 gennaio 1978, fra lo Stato Spagnolo e la Santa Sede, su materie giuridiche, dove si dice che „lo Stato riconosce gli effetti civili al matrimonio celebrato a seconda delle norme del Diritto canonico", e che "gli effetti civili del matrimonio canonico si producono dal momento della celebrazione".

Invece, larticolo 7.1 dell'accordo di cooperazione (o intesa) dello Stato colla Commissione Islamica di Spagna (CIE) dice semplicemente che „si attribuiscono effetti civili al matrimonio celebrato colla forma religiosa stabilita nella Legge Islamica, dal momento della sua celebrazione, sempre che i contraenti abbiano compiuto i requisiti di capacità richiesti nel Codice civile”.

Riassumendo: in Spagna, una persona sposata e non divorziata non potrà fare un secondo matrimonio, o terzo, ecc. (caso di poligamia successiva), nè contrarre con più d'una persona allo stesso tempo, perchè ambedue sono situazioni che contravengono le norme di capacità matrimoniale del Diritto civile spagnolo.

\section{LA PRotezione della monogamia in Spagna}

a) I divieti di contrarre nuovo matrimonio nella legislazione civile e canonica

Nella legislazione civile, le norme sulla capacità matrimoniale a cui abbiamo già fatto riferimento sono l'articolo 46.2 del Codice civile, che recita che „non possono contrarre matrimonio (...) quelli che si trovano legati con vincolo matrimoniale", e l'articolo 47.3 dove si dice che "neanche possono contrarre matrimonio tra di loro (...) i condenati come autori o complici della norte dolosoa del coniuge di qualsiasi di loro".

Inoltre, se parliamo della legislazione canonica, si vede che i divieti civili si corrispondono con gli impedimenti canonici di vincolo e di crimine.

Così, il canone 1085.1, che regola l'impedimento di vincolo, stabilisce che „attenta inválidamente il matrimonio chi si trova legato per il vincolo di un matrimonio precedente, benchè non sia stato consommato", e nel comma 2 , che „ancorchè il matrimonio precedente sia nullo o abbia stato dissolto per qualsiasi motivo, no per questo è lecito contrarre un altro prima di chè si 
sia constanz legítimamente e con certeza la nullità o dissoluzione del precedente".

Il fondamento di questo impedimento si trova nella proprietà dell'unità del matrimonio, ossia la monogamia, non quella dell'indissolubilità. Anzi, questo impedimento viene considerato come un divieto matrimoniale di diritto naturale, e per questo, non si può dispensare da nessuna autorità nella Chiesa cattolica. È per questo che il diritto matrimoniale canonico non ammette il divorzio, ma questo ci porterebbe a un altri aspetti al di fuori dello scopo di queste riflessioni.

'E chiaro che tanto il Diritto civile come quello canonico hanno la previssione normativa che vieta la celebrazione di ulteriori matrimoni, per proteggere la monogamia.

D’altra parte, l'impedimento canonico di crimine, regolato nel canone 1090, contiene due modalità: il congiugicidio individuale, nel suo comma primo, ed il congiugicidio con cooperazione mutua (detto „utroque machinante”), nel comma secondo. Così, in primo luogo dice che „chi, per contrarre matrimonio con una persona determinata, provoca la morte del coniuge d'essa, o del suo stesso coniuge, attenta inválidamente il matrimonio", e poi, dice che „anche attentano inválidamente il matrimonio tra di loro, loro cjhe con mutua cooperazione, física o morale, provocarono la norte del congiuge".

Evidentemente, questo impedimento canonico, come il suo corrispondente civile, hanno lo scopo di impediré che sia possibile liberarsi del divieto di contrarre matrimonio per essere legato con un vincolo matrimoniale provocando la morte del congiuge, dato che con la morte si toglierebbe il vincolo matrimoniale e, in conseguenza, anche il divieto per contrarre di nuovo.

Finalmente, le norme spagnole su riagruppamento familiare sono anche protettive della monogamia: in modo molto breve dobbiamo dire che nel caso in cui ci sia un migrante polígamo, soltanto si può fare la riagruppazione familiare con una delle sue mogli, non con tutte le altre che possa avere.

b) Il reato di bigamia

Al di fuori del diritto civile spagnolo, la protezione della monogamia si trova anche nel diritto penale, col reato di bigamia. Così, la bigamia viene penalizzata nell'articolo 217 del Codice Penale, che dice che „chi faccia un 
secondo od ulteriore matrimnio, sapendo che il matrimonio precedente sussiste legalmente, sarà punito con la pena di prigione di sei mesi ad un anno".

La bigamia viene tipificata non solo per contrarre un secondo matrimonio, ma anche per gli ulteriori matrimoni poligamici: terzo (trigamia) e quarto (tetragamia), che sono le possibilità ammesse dal diritto Islamico, sempre che l'uomo possa mantenere tante mogli.

Abbiamo già parlato sull'origine medievale del reato di bigamia nei diritti occidental come quello spagnolo. Dobbiamo aggiungere adesso che il reato di bigamia, nel suo origine, non aveva lo scopo di proteggere l'unità del matrimonio, vale a dire la monogamia, ma lo stato civile delle persone. La protezione della monogamia come garantía dell'unità matrimoniale arrivò, col tempo, grazie al cristianesimo, ed anzi, per la sua connessione coll'indissolubilità del matrimonio canonico la bigamia si applicò anche a tutti i comportamenti divorzisti che potrebbero attaccare l'unità matrimoniale. Per questo si parlava di bigamia simultanea, che è il vero attacco a l'unità, e di bigamia successiva, che in realtà proteggeva l'indissolubilità (Vega Gutiérrez, 1997, p. 520). Tuttavia, il modo in cui si arrivi a una situazione di coesistenza di più di un vincolo matrimoniale, sempre che ci sia un secondo o ulteriori matrimoni cè bigamia.

Nel diritto spagnolo odierno, a seconda dell'articolo 219.1 del Codice Penale, il reato di bigamia non solo può essere commesso da chi sia il contraente poligámico, ma anche dal ministro di culto che lo permetta e autorizzi la sua realizzazione, come cooperatore necessario, in questo caso, un imano o dirigente religioso islámico, a seconda dell'articolo 3.1 dell'accordo di cooperacione con i musulmani. Questa è la interpretazione del contenuto di questo articolo, che essattamente dice che „chi faccia l'utorizzazione di un matrimonio dove ci sia una causa di nullità conosciuta (come sarebbe, infatti, la sussistenza di un vincolo matrimoniale precedente) o dinunziata nel procedimento matrimoniale previo (chiamato in spagnolo „expediente matrimonial"), sarà punito con la pena di prigione di sei mesi a due anni ed inabilitazione speziale per lavoro o carico pubblico di due a sei anni”.

Nonnostante questa previsione penale per punire la bigamia, la legislazione ecclesiastica spagnola non ha previsto in modo adeguato che non ci siano 
matrimoni poligamici fatti, diciamolo cosí, „di buona fede”, per persone musulmane che pensino che in Spagna l'ordine pubblico sia come quello dei paesi musulmani e per questo, sarebbero ammessi dei matrimoni poligamici, sebbene, non rendendosi conto del fatto che l'ignoranza del diritto non è scusa valida per non attuare d'accordo colla legge, e che queste possibilità sia impensabile che possa darsi negli imani e ministri di culto musulmani che autorizzino un matrimonio poligámico, perchè è da supporre che questi devono avere una mínima conoscenza delle norme del paese dove esercitano il suo ministerio religioso, il cui, anche, ha delle conseguenze giuridiche come il mutamento dello stato matrimoniale delle persone.

Questa situazione è una conseguenza della mancata previsione nella legge spagnola, perchènon cè un controllo previo alla celebrazione del matrimonio islámico de parte di nessuna autorità spagnola. In questo senso, l'articolo 7 dell'Intesa coi musulmani spagnoli soltanto prevede un controllo a posteriori della celebrazione del matrimonio, nel momento della sua iscrizione (comma due e comma tre): „2. Le persone che desiderino iscrivere il matrimonio celebrato nella forma prevista nel numero anteriore (il quale vuol dire la forma prevista dalla legge islámica), dovranno accreditare previamente la sua capacità matrimoniale per mezzo di una certificazione agevolata dal Registro Civil corrispondente. Non potrà farsi l'iscrizione se si celebra il matrimonio dopa il trascorso di sei mesi dall'espedizione di questa certificazione”. / „3. Una volta celebrato il matrimonio, il rappresentante della Comunità Islamica dove si abbia celebrato il matrimonio, spedirà al Registro Civil, per la sua iscrizione, una certificazione dove ci sia accreditata la celebrazione del matrimonio e dove ci sia accreditata la celebrazione del matrimonio, e dove ci saranno menzionate le circostanze che exige la legislazione del Registro Civil”.

$\mathrm{E}$, come si è già detto, questo può accadere (la celebrazione al meno di fatto, di un matrimonio poligamico), perchè la poligamia è ammessa dalla Legge Islamica. Infatti, il comma primo dell'articolo 7 dell'accordo di cooperazione recita: „Si attribuiscono effetti civili al matrimonio celebrato a seconda della forma stabilita nella Legge islámica, dal momento della sua celebrazione, se i contraenti hanno dei requisiti di capacità stailiti nel Código Civil. / I contraenti esprimiranno il consenso davanti di qualcuna delle persone 
dette nel numero 1 dell'articolo 3 (vale a dire, i dirigenti religiosi islamici e gli imam delle comunità Islamiche), e. almeno, due testimoni maggiorenni. / Per il pieno riconoscimento di tali effetti, si vuole l'iscrizione del matrimonio nel Registro Civil”.

\section{LA POLIGAMIA ISLAMICA}

a) Fondamento e caracteristiche.

Infatti, il diritto musulmano ammete la poligamia nella sua versione di poliginia, a seconda della quale un varone sposa parecchie donne (fa lo stesso che sia in modo successivo o nello stesso atto, fino a quattro, sempre che possa mantenere loro). Ė noto che la Legge Islamica ammette la tetragamia per l'uomo, ma impone la monogamia allá donna (Bonet Navarro e Vento Torres, 1994, p. 829; Combalia, 2001, p. 15). Veramente non cè un obbligo, perchè la poligamia è ammessa come soluzione al problema derivato di ci essere più donne che unomini nella società musulmana.

Il testo del Corano che fa allusione allá poligamia si trova nel capitolo IV, verso 3, che recita: „Se avete paura di non essere equi coi orfani, sposate le donne che vi piacciano: due, tre o quattro. Se avete paura di non essere giusti, sposate soltanto una" (Olmos Ortega, 2008, p. 498).

Almeno per i sciiti (Muttahari, 1988, p. 156) la poligamia è considerata come un diritto delle donne, il cui consistirebbe nel fatto di avere la possibilità di avere sempre al meno uno sposo, sebbene questo sposo sia compartito con altre donne che siano allo stesso tempo spose di quel'uomo, dato che questa situazione sarebbe migliore che non avere nessuno.

Per spiegare che questa situazione, che contrasta tanto con la visione occidentale della stessa (dove tutt'altro che un diritto della donna, la poligamia è una situazione di desuguaglianza tra le persone per ragione del suo sesso che, come abbiamo visto, motiva la sua esclusione dell'ordinamento giuridico spagnolo per ragioni d'ordine pubblico) si trova in due motivi allacciati. D'una parte, il fatto che l'Islam considera che lo stato naturale delle persone adulte (uomini e donne) è quello di essere sposati, perchè nell'Islam non si concepisce la vita celibataria o „religiosa” come accade nel cristianesimo. 
D'altra parte, come secondo motivo per ammettere la poligamia, si debe avere conto del fatto che la religione islámica, sin dalla sua nascita nell'Arabia, si allargò territorialmente in pocchi anni per vasti territori dell'Asia e dell'Afica settentrionale per mezzo della guerra. La conseguenza delle guerre d'espansione della religione fu che la quantità di uomini „disponibili” per il matrimonio calò, per il fatto che allá guerra soltanto andavano gli uomini, dove molti di loro imorivano, in tanto che il ruolo domestico delle donne risparmiò loro del rischio di morire. In conseguenza, la poligamia risolve il problema di sposare tante donne in una società dove ci sono molte più donne che uomini.disponibili per il matrimonio (Bonet Navarro, 1994, pp. 479-480).

Finalmente, la poligamia servirebbe anche per riparate l'infertilità della prima moglie, come accade normalmente, o d'una ulteriore moglie, che il marito non vuole ripudiare. In questo caso, la spiegazione si trova nella concezione patriarcale del matrimonio e della familia musulmana, per cui è molto importante avere un fliglio maschio, tanto di essere in molti casi lo stesso motivo del matrimonio.

c) La sentenza della Corte Suprema Spagnola di febbraio 2018

Nonnostante tutto quello che abbiamo detto finora sulla poligamia musulmana e la sua interdizione dell'ordinamento giuridico spagnolo, il vero è che l'ordine pubblico non debe supporre sempre, in tutti i casi, un muro impossibile di superare, perchè sempre debe considerarsi una certa flessibilità in alcuni casi concreti negli scopi della giustizia.

In questo senso, la Sala de lo Contencioso-Administrativo dello Tribunal Supremo spagnolo (Corte Suprema di Giustizia di Spagna), il 15 gennaio 2018, riconoscette il diritto allá pensione di vedovanza alle due mogli di un militare marocchino polígamo, che aveva laborato molto tempo fa, negli anni settanta dello scorso secolo, alle ordini dell'esercito spagnolo, purtroppo questa prattica matrimoniale sia contraria all'ordine pubblico spagnolo.

Nel caso concreto a cui facciamo riferimento, la Corte Suprema spagnola si è pronunciato in modo affermativo allo ricorso di casazione presentato dalla seconda moglie del militare morto contra la decisione (sentencia) della Corte inferiore (che in questo caso era il Tribunal Superior de Justicia 
de Madrid), che aveva respinto la pensione di vedovanza chiesta da questa donna, pensione che soltanto si aveva riconosciuto allá prima moglie. La decisione della Corte Suprema non supone radoppiare la pensione, ma che sia divisa fra el due moglie (o meglio, già vedove).

La Sala de la Corte Suprema non nega la sua dottrina nel senso che la poligamia sia contraria allordine pubblico spagnolo, ma precisa che questo criterio non può applicarsi in questo caso concreto perchè è stato il proprio Stato spagnolo chi ammise (tanti anni fa) un determinato effetto a tale matrimonio nell'articolo 23 del Convegno di Sicurezza Sociale firmato tra la Spagna e il Marocco nel 1979 (momento in cui la Costituzione spagnola attuale era già in vigore). E il testo di questa norma dice che „la pensioen di vedovanza causata per un operaio marrocchino sarà divisa, nel suo caso, per parti iguali, tra chi sia, a seconda della legislazione marrocchina, beneficiaria di questa pensione". In realtà, se non ci fosse questo compromesso legale previo fatto dallo Stato Spagnolo in quel Convengo, è da supporre che non si avrebbe pottuto ammettere una domanda del genere. Anzí, nella sua decisione, la Corte Suprema spagnola non fa altro che interpretare che la condizione di „beneficiaria della pensione” si può estendere a tutte le donne (mogli) sposate simultáneamente col operaio, in una situazione di poligamia, e che la situazione di poligamia non vieta, per motivi dordine pubblico il riconoscimento del diritto allá pensione di vedovanza a favore di tutte le mogli chi, d'accordo con la sua legge personale, era sposate simultaneamente col operaio morto.

A questo scopo, si deve capire che la simultaneità matrimoniale si riferisce al momento della morte dello sposo, in relazione con le mogli che aveva in quel momento, indipendentemente del momento concreto in cui si siano sposate ognuna di loro con lui. In altre parole, non c'entra niente che tutte siano sposate allo stesso tempo che in modo graduale, cosa che, dall'altro è il modo più abituale di arrivare a un matrimonio poligamico.

Come conseguenza, la pensione di vedovanza verrà divisa fra le vedove, in modo che le parti corrispondenti a ognuna di loro, tutte sommate, sia il totale di una sola pensione di vedovanza. Questa divisione può farsi in modo che ognuna delle vedove abbiano la stessa quantità, o fare un riparto proporzionale a seconda del tempo di convivenza (Olmos Ortega, 2008, p. 507). 
Questa soluzione, che supone una figura indebolita dellordine pubblico spagnolo, quello detta come „orden público atenuado” (Olmos Ortega, 2088, pp. 506) e già data nel passato in decisioni di livelli giudiciari inferiori, come quella del Tribunal Superior de Justicia di Galicia del 2 aprile 2002, che faceva riferimento a un operaio senegalese morto in un incidente stradale che aveva contratto matrimonio con due donne a seconda della sua legge nazionale, e tutte e due vedove chiederono la corrispondente pensione di vedovanza (Olmos Ortega, 2008, p. 507).

\section{RIFLESSIONE CONCLUSIVA}

La società spagnola si trasforma, pian piano, in una società ogni volta più multiculturale, dove possono coessistere differenti modi di intendere la famiglia ed il matrimonio a seconda de ogni religione, sempre che siano conciliabili con l'ordine pubblico spagnolo, dove sono garantiti i diritti umani, la dignità delle persone e l'uguaglianza tra di loro. La poligamia si trova in evidente contrasto con l'ordine pubblico spagnolo per la situazione di disuguglianza delle donne sposate in un matrimonio del genere. Per questo, sono in grado di assicurare che la poligamia non sarà mai ammessa in Spagna come una situazione matrimoniale valida in tutta la sua pienezza. I casi ricenti di decisioni della giurisprudenza spagnola in cui sono ammessi degli effetti giuridici concreti, in materia di pensione di vedovanza, a mio avviso, sono soltanto casi molto concreti in cui, agli scopi di evitare situazioni ingiuste, lordine pubblico attua in modo indebolito o atenuato, ma mantenendo il suo rigore per le altre situazioni dove dalla sua applicazione non derivano situazioni di ingiustizia, ed anche, (nell'unico caso in cui si sono ammesse delle conseguenze di un matrimonio poligámico), per compiere dei compromessi legali precedenti. 


\section{Literatura}

Bočáková, O., Kubíčková, D. (2015). Rodzina i jej pozycja w nowym tysiącleciu. Journal of Modern Science, 26(3), 33-46. ISSN 1734-2031.

Bonet Navarro, J. (1994). El matrimonio en el derecho islámico, en Curso de Derecho Matrimonial y Procesal Canónico para profesionales del foro XI, Salamanca: Universidad Pontificia de Salamanca, pp. 467-481.

Bonet Navarro, J. e Vento Torres, M. (1994). El islamismo, en Acuerdos del Estado español con los judíos, musulmanes y protestantes, Salamanca: Universidad Pontificia de Salamanca, pp. 71-94.

Chehata, C. (1974). El vínculo matrimonial en el Islam, en Matrimonio y divorcio, Salamanca: Sígueme, pp. 59-72.

Combalia, Z. (2001). Estatuto de la mujer en el derecho matrimonial islámico, en Revista Aequalitas 6, pp. 15-20.

Combalia, Z. (2005). Inmigración y matrimonio en España, en Cuestiones actuales de Derecho Canónico y Eclesiástico en el XXV Aniversario de los Acuerdos con la Santa Sede y XX Aniversario de la vigencia del CIC, Salamanca: Publicaciones Universidad Pontificia de Salamanca, pp. 283-301.

Gaudemet, J. (1993). El matrimonio en Occidente, Madrid: Taurus.

Krajewska A. (2013) Zagrożenia rodziny $w$ dobie kryzysu w: Such-Pyrgiel M. (red) Bezpieczeństwo w XXI wieku w ujęciu socjologicznym, pedagogicznym, prawnym i nauk o zarządzaniu. Józefów: Wydawnictwo WSGE. ISBN 978-83-63753-37-6, s. $105-116$.

Morán García, M. E. (2000). La familia multicultural. Entre el conflicto de civilizaciones y la paz entre los pueblos, en Estudios en homenaje al Prof. Martínez Valls, 2, Alicante: Universidad de Alicante, pp. 843-860.

Moreno Antón, M. (2001). El matrimonio islámico ante el Derecho español, en Derecho de familia y libertad de conciencia en los países de la Unión Europea y el Derecho comparado, Bilbao: Universidad del País Vasco, pp. 619-630.

Motilla, A. e Lorenzo, P. (2012). Derecho de familia islámico, Madrid: Colex.

Muttahari, S. (1988). I diritti della donna nell'slam, Roma: Centro Culturale Islamico.

Navarro-Valls, R. (1994), Matrimonio y Derecho, Madrid: Tecnos.

Olmos Ortega, M. E. (2008), Mujer, matrimonio e Islam, en Anuario de Derecho Eclesiástico del Estado 25, pp. 493-523. 
Olmos Ortega, M. E. e Landete Casas, J. (2013), Legislación eclesiástica, 25 ed., Pamplona: Civitas.

Prader, J. (1986). Il matrimonio nel mondo. Celebrazione. Nullità e scioglimento del vincolo, Milano: Cedam.

Rodríguez Chacón, R. (1994). El matrimonio religioso no canónico en el derecho español, en Anuario de Derecho Eclesiástico del Estado 10, pp. 371-383.

Rodríguez García, J. A. (2001). Poligamia: libertad religiosa y discriminación de la mujer, en Derecho de familia y libertad de conciencia en los países de la Unión Europea y el Derecho comparado, Bilbao: Universidad del País Vasco, pp. 745-760.

Rodríguez Marcos, M. E. (2006). Familias interculturales: la construcción de la interculturalidad de los micro social a lo macro social, Salamanca: Universidad Pontificia de Salamanca.

Ruano Espina. L. (2003). Derecho e Islam en España, en Ius Canonicum 42, pp. 465-543.

Such-Pyrgiel M. (2012). Rodzina i jakość życia we współczesnym społeczeństwie polskim. Zarys problematyki w: Sitek M., Such-Pyrgiel M., Przeastek D. (red) Bezpieczeństwo i jakość życia we współczesnym społeczeństwie polskim. Studium interdyscyplinarne, Józefów: Wydawnictwo WSGE. ISBN 978-83-63753-62-8, s. 13-33.

Vega Gutiérrez, A. M. (1997), La unidad matrimonial y su tutela penal: precedentes romanos y canónicos del delito de bigamia, Granada: Comares. 